\title{
Incipient failure in sandpile models
}

\author{
Onuttom Narayan \\ Department of Physics, University of California, Santa Cruz, CA 95064 \\ Sidney R. Nagel \\ The James Franck Institute and Department of Physics, University of Chicago, Chicago, IL 60637
}

(December 5, 2017)

\begin{abstract}
Elastoplastic and constitutive equation theories are two approaches based on very different assumptions for creating a continuum theory for the stress distributions in a static sandpile. Both models produce the same surprising prediction that in a two dimensional granular pile constructed at its angle of repose, the outside wedge will be on the verge of failure. We show how these predictions can be tested experimentally.
\end{abstract}

\section{Introduction}

Understanding the stress distributions in static piles is a basic and open question in the field of granular materials. If one tries to construct a continuum coarse-grained description of such systems, the difficulty is that, unlike for elastic materials, there is no obvious relation connecting the stresses to any strain fields. In two dimensional systems, there are three variables, the components of the symmetric stress tensor, but only two force balance equations in the two orthogonal directions. If the three components of the stress tensor can no longer be expressed in terms of the two components of a displacement field, one needs one additional equation to obtain the stresses. In general three dimensional systems, the situation is even worse: one has six components of the stress tensor instead of three components of a displacement field, so that there are three 'missing' equations.

One approach for understanding the general structure of the stress distributions in granular systems is in terms of a grain by grain statistical method [1]. However, one would hope that, on length scales much larger than the individual grain size, it should be possible to construct a continuum theory for a static pile of granular matter (a "sandpile"). Several very different approaches for constructing such a theory have been tried. Two particularly interesting recent models are the elastoplastic theories epitomized in the work of Cantelaube and Goddard [2] and the constitutive equation models introduced by Bouchaud et al. [3], and subsequently generalized by Wittmer et al. [4]. Despite their very different assumptions, both approaches obtain very similar results for the stress distribution in a two-dimensional triangular sandpile obtained by pouring grains from a point source. In particular, they predict that for such a sandpile there is a wide wedge of material near the surface of the pile that is on the threshold of failure, in that it is unstable to the application of further shear stress.

On the face of it, the result of a large wedge at the instability threshold predicted by both approaches is sur- prising since there is a great deal of experience with the flow caused by tilting a pile at its angle of repose. In such avalanches the motion is confined only to grains in a thin boundary layer near the surface [5] [6]. From such observations one might have naively expected that only the surface of the sandpile, and not an entire wedge, would be unstable.

These are dramatic predictions that are inescapable consequences of the assumptions of these two types of theories. It is important to see if experiments can be designed to detect the unstable regions. The purpose of the present paper is to explore the general consequences of having a wedge of plastic material near the surface of the pile, and show what experiments can be performed to probe its existence.

In the constitutive equation models [3] [4] it is suggested that the components of the stress tensor are actually related to one another. As mentioned above, in two dimensions one needs one such "constitutive equation" interrelating the stresses; in general three-dimensional systems one needs three constitutive equations, which can be reduced in systems with special symmetry. Although the constitutive equations in general depend on how the system is assembled, the hypothesis that these equations are local implies that they are determined in any part of the pile when that part is constructed, and are not affected by subsequent changes in the loading or by rearrangements in other regions of the pile. Focusing on a two dimensional sandpile that is built by pouring grains from a point source, through scaling and symmetry arguments, Wittmer et al. [1] are led to consider constitutive equations that impose a linear relationship among the stress components. One is ultimately left with a one-parameter family of models and solutions thereof.

For a two dimensional sandpile at its angle of repose, all the models in the one-parameter family obtain stresses that are linear functions of the spatial coordinates, and predict that outside a symmetric triangular "inner region", the entire pile is on the verge of failure. This is in 
the sense that, if $\phi$ is the angle of repose, and $r$ and $z$ are the coordinate directions as shown in Fig. 1, the stresses in the outer region satisfy the condition

$$
\frac{\left(\sigma_{r r}-\sigma_{z z}\right)^{2}+4 \sigma_{r z}^{2}}{\left(\sigma_{r r}+\sigma_{z z}\right)^{2}}=\sin ^{2} \phi
$$

i.e. the Mohr-Coulomb criterion is saturated. This is equivalent to the statement that, at any point in the outer region, one can find a suitably oriented plane for which the ratio of the shear stress to the normal stress is $\tan \phi$, which is the maximum possible value before plastic flow takes place.

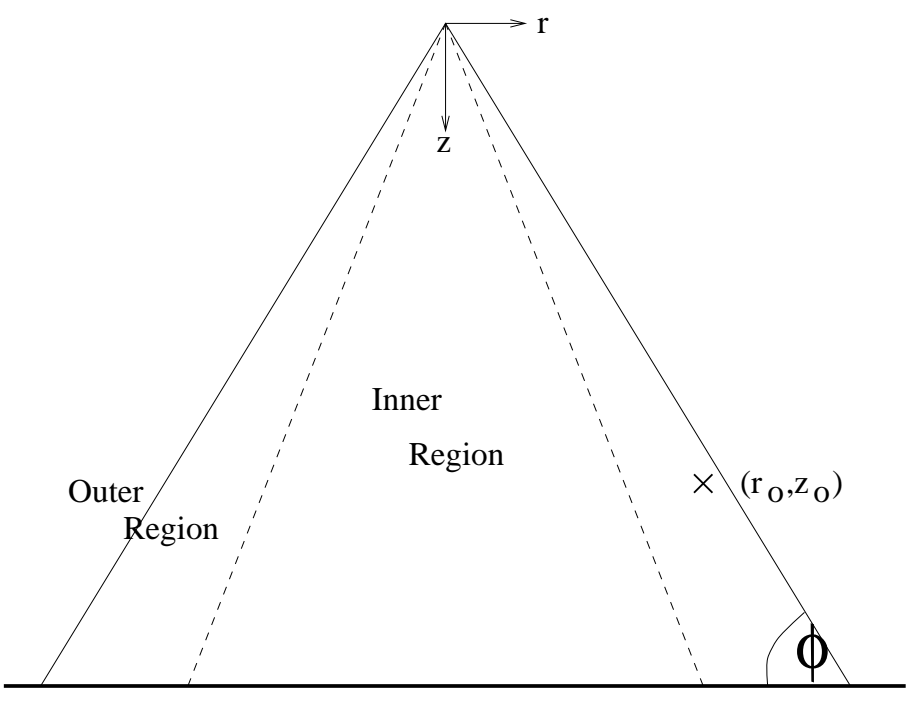

FIG. 1. Two dimensional sandpile at its angle of repose. The point $\left(r_{0}, z_{0}\right)$ will be used later in the paper.

The stress distribution obtained by Cantelaube and Goddard [2] is similar to that obtained by Refs. [3] and [ [4], although these authors start from a completely different point of view. They assume that a granular material can be treated as an elastic medium, with the added feature that when the ratio of the shear strain to the normal strain goes beyond a critical value, the corresponding ratio of stresses saturates at $\tan \phi$. Thus a sandpile can be divided into elastic and plastic regions, with the stresses expressible in terms of displacement fields in the elastic regions, and satisfying the MohrCoulomb yield criterion in the plastic regions. For a two-dimensional system, one can eliminate the strains from the problem and obtain expressions entirely in terms of stresses. (One still needs to specify the boundaries between the elastic and plastic regions in order to determine the stresses.) If one assumes a scaling form $\sigma_{i j}=z f_{i j}(r / z)$ for the stresses, one finds that the region adjacent to the sandpile surface must be plastic. Thus the elastoplastic models [2] and the ones proposed by Bouchaud et al. [3] and Wittmer et al. [4] are in agreement in predicting a wide wedge of material near the surface of a sandpile at its angle of repose that is on the threshold of failure. However a surprising, and we think counterintuitive, result of the elastoplastic approach [2] is that even for a triangular sandpile with arbitrary slope including one well below its angle of repose, the plastic region must persist if the stresses satisfy the scaling form above.

In the rest of this paper, we find the destabilizing perturbations that can experimentally probe the plastic region in these two types of theories. Because their approaches are different, they must be considered separately. In the scenario of Refs. [3] and [4], an appropriately chosen infinitesimal perturbation anywhere in the outer region of the sandpile destabilizes it. We show this by demonstrating that, when the extra perturbation is included, there is no solution to the equations for the stresses that satisfies the Mohr-Coulomb yield criterion everywhere in the pile. The effects of such a perturbation necessarily extend to the outer surface of the pile. For elastoplastic theories, an infinitesimal force applied in an appropriate direction to a section of the bottom of the plastic part of the pile is sufficient to destabilize it. We discuss how these results would be modified for a material with a non-zero Bagnold hysteresis angle.

\section{Constitutive equation models}

In the scenario of Refs. [3] and [4], if a localized body force of magnitude $f$ is applied in the positive $r$ direction at some point $\left(r_{0}, z_{0}\right)$ in the outer region (see Fig. 11), the equations satisfied by the change $\sigma^{\prime}$ in the stress tensor $\sigma$ are

$$
\begin{aligned}
& \partial_{r} \sigma_{r r}^{\prime}+\partial_{z} \sigma_{r z}^{\prime}=f \delta\left(r-r_{0}\right) \delta\left(z-z_{0}\right) \\
& \partial_{r} \sigma_{r z}^{\prime}+\partial_{z} \sigma_{z z}^{\prime}=0
\end{aligned}
$$

with the boundary conditions that the change in the normal and shear stress on the sloping sidewalls of the sandpile must be zero. As discussed in the previous section, the arguments suggesting a local constitutive equation imply that this equation is not affected by subsequent changes in the loading, so that the components of the change in the stress tensor must also satisfy the equation

$$
\sigma_{r r}^{\prime}=\eta \sigma_{z z}^{\prime}+\mu \sigma_{r z}^{\prime}
$$

where $\eta$ and $\mu$ are related, so that there is one free parameter in the equations. [4 For $\mu \neq 0$, Eq.(3) is actually two different equations on both sides of the symmetry axis $r=0$, leading to a kink in the stress tensor. We shall ignore this at present, since we shall later see that it is of no consequence.

From Eq. (2]b), $\sigma_{z z}^{\prime}=-\partial_{r} V$ and $\sigma_{r z}^{\prime}=\partial_{z} V$, where $V$ is a scalar field. Substituting in Eq.(2a), we obtain

$$
-\eta \partial_{r}^{2} V+\mu \partial_{r} \partial_{z} V+\partial_{z}^{2} V=\delta\left(r-r_{0}\right) \delta\left(z-z_{0}\right) f .
$$

The homogenous version of this equation is an asymmetric wave equation [4] with wave velocities $d r / d z=c_{1}$ and $-\left|c_{2}\right|$. Eq.(1) has the solution 


$$
\begin{aligned}
V(r, z)=\lambda\left[\Theta\left(c_{1}\left(z-z_{0}\right)-r+r_{0}\right)\right. & \\
& \left.-\Theta\left(\left|c_{2}\right|\left(z_{0}-z\right)-r+r_{0}\right)\right]
\end{aligned}
$$

with $\lambda=f /\left(c_{1}+\left|c_{2}\right|\right)$. Therefore we obtain

$\sigma_{z z}^{\prime}=\lambda\left[\delta\left(c_{1}\left(z-z_{0}\right)-r+r_{0}\right)-\delta\left(\left|c_{2}\right|\left(z_{0}-z\right)-r+r_{0}\right)\right]$

$\sigma_{z r}^{\prime}=\lambda\left[c_{1} \delta\left(c_{1}\left(z-z_{0}\right)-r+r_{0}\right)+\right.$

$$
\left.\left|c_{2}\right| \delta\left(\left|c_{2}\right|\left(z_{0}-z\right)-r+r_{0}\right)\right]
$$

$\sigma_{r r}^{\prime}=\eta \sigma_{z z}^{\prime}+\mu \sigma_{r z}^{\prime}$.

All components of the change in the stress tensor thus consist of a pulse travelling outward at a velocity $c_{1}$ and another travelling inward with a velocity $c_{2}$, as one proceeds downward into the pile. This is as one would expect for a wave equation. The inward pulse is partly reflected and partly refracted when it reaches the symmetry axis $r=0$, emerging as two outward pulses both with speed $c_{1}$.

Since we wish to demonstrate a violation of the MohrCoulomb criterion, it is sufficient to do this for one of the pulses. Accordingly, we restrict our attention to the path of the outward propagating pulse. Along this path, we have

$$
\begin{aligned}
& \sigma_{z z}^{\prime}=\sigma_{z r}^{\prime} / c_{1} \\
& \sigma_{r r}^{\prime}=c_{1} \sigma_{z r}^{\prime}
\end{aligned}
$$

with $\sigma_{z r}^{\prime}>0$ for $f>0$. In the last of these equations, we have used the relation [4] $\eta / c_{1}+\mu=c_{1}$ which is valid for all $\mu$. When $\sigma^{\prime}$ is small [7], the requirement that the Mohr-Coulomb criterion should be satisfied is equivalent to the condition

$$
\frac{\left(\sigma_{z z}-\sigma_{r r}\right)\left(\sigma_{z z}^{\prime}-\sigma_{r r}^{\prime}\right)+4 \sigma_{r z} \sigma_{r z}^{\prime}}{\left(\sigma_{z z}+\sigma_{r r}\right)\left(\sigma_{z z}^{\prime}+\sigma_{r r}^{\prime}\right)} \leq \sin ^{2} \phi .
$$

One can verify that, irrespective of $\mu$, the unperturbed stresses satisfy the conditions $4\left(\sigma_{z z}-\sigma_{r r}\right)=$ $2 \sigma_{r z} \tan \phi=\sin ^{2} \phi\left(\sigma_{z z}+\sigma_{r r}\right)$. Substituting in Eq.(8), the inequality simplifies to

$$
\sigma_{r z}^{\prime}-(\tan \phi) \sigma_{r r}^{\prime} \leq 0
$$

which from Eq.(7) is equivalent to

$$
\left[1-c_{1} \tan \phi\right] \sigma_{z r}^{\prime} \leq 0 .
$$

Since $\sigma_{z r}^{\prime}>0$ for $f>0$, and $c_{1} \tan \phi<1$ in order for the existence of an outer region in the sandpile, we see that a horizontal outward directed force added anywhere in the outer region of the sandpile results in there being no possible solution to the stress equations, i.e. the sandpile is destabilized.

\section{Elastoplastic models}

In the scenario of Ref. [2], one assumes that the sandpile can be divided into elastic regions, where the stresses are related to underlying strain fields, and plastic regions where Eq.(1i) is satisfied. The specific solution for a sandpile at its angle of repose in Ref. [2] has an outermost plastic region in which the stresses are linear functions of the spatial coordinates. This is, in fact, a general consequence of assuming a scaling form $\sigma_{i j}=z f_{i j}(r / z)$ for the stresses, valid even when the angle of the sandpile is below the angle of repose. Since this is not proved in Ref. [2], we outline the proof here.

Choosing units in which the gravitational force per unit volume $\rho g$ is unity, the force balance equations for the pile are

$$
\begin{aligned}
\partial_{r} \sigma_{r r}+\partial_{z} \sigma_{r z} & =0 \\
\partial_{r} \sigma_{r z}+\partial_{z} \sigma_{z z} & =1 .
\end{aligned}
$$

With a scaling form for the stresses, these yield

$$
\begin{aligned}
& f_{r r}^{\prime}+f_{r z}-S f_{r z}^{\prime}=0 \\
& f_{r z}^{\prime}+f_{z z}-S f_{z z}^{\prime}=1
\end{aligned}
$$

where $S=r / z$ is the argument of the scaling functions and the prime indicates differentiation with respect to $S$. In an elastic region, the fact that the stresses are related to underlying strain fields requires the consistency condition

$$
\partial_{z} \partial_{z} \sigma_{r r}+\partial_{r} \partial_{r} \sigma_{z z}=\partial_{r} \partial_{z} \sigma_{r z}
$$

which with the scaling form for the stresses is equivalent to

$$
S^{2} f_{r r}^{\prime \prime}+f_{z z}^{\prime \prime}=-S f_{r z}^{\prime \prime} .
$$

Differentiating Eqs.(12), together with Eq.(14), we have three linear homogenous equations in the three second derivatives $f_{i j}^{\prime \prime}$. Since the condition for the three equations to be linearly dependent is $1+S^{2}+S^{4}=0$, which is not satisfied for any real $S$, it follows that $f_{i j}^{\prime \prime}$ must all vanish, i.e. the stresses in any elastic region are linear functions of the spatial coordinates. Since all the stresses must vanish on the outer surface of the sandpile, it follows that if the outermost region were elastic, the stresses would be of the form $\sigma_{i j}=k_{i j} z\left(1-S / S_{0}\right)$, where $r / z=S_{0}$ on the outer surface of the sandpile. Thus the ratios of the stresses to each other are independent of spatial coordinates in this region. From Eq.(1), it is then clear that if the Mohr-Coulomb criterion is satisfied as an inequality (as it must inside an elastic region), it will be satisfied as an inequality even on the inner boundary of the region, so that one cannot patch the solution to a plastic region. Since it is impossible 22 to obtain a fully elastic solution for the stresses in a sandpile, the outermost region must be plastic. 
In a plastic region, Eq.(14) will not be satisfied. However, Eqs. 12) are still valid. For the outermost region, this implies that the scaling functions $f_{i j}(S)$ are all linear in $S-S_{0}$ close to the surface of the pile. The proportionality constants can be obtained from Eq.(11) with Eqs.(12). But since Eqs.(12) with the derivative of Eq.(11) constitutes a system of first order differential equations in the scaling functions $f_{i j}$, one can evolve them to obtain the stresses everywhere in the outermost plastic region once they are known at the surface. Since choosing $f_{i j}$ to be a linear function of $S-S_{0}$ can be seen to be a valid solution not just close to the surface of the pile, but even as one proceeds into its interior, it follows that this is the correct solution.

Thus we see that for any angle for the sandpile, the outermost region must be plastic, and the stresses are linear homogenous functions of $\left(z-r / S_{0}\right)$. The extent of this plastic wedge is a free parameter in elastoplastic theories, partly constrained by the requirement of matching to an elastic region. Since the ratios of the stresses to each other are independent of location in the plastic zone, it follows that the yield lines, along which the system destabilizes under infinitesimal extra shear, have the same orientation everywhere in the outermost plastic zone. For the case when the pile is at the angle of repose, the yield lines are vertical and along the surface of the pile. If the plate supporting it at the bottom is made of segments, and an infinitesimal extra upward force is applied to a segment below the plastic region of the pile, the segment will move upwards. If the extra force is kept constant, independent of the displacement of the segment, the sandpile will not be able to resist the force, and the segment will continue to move upwards, resulting in overflow at the top surface. This is in contrast to the response that one would obtain in the elastic regions, where an infinitesimal extra applied force would cause a slight upward motion, at which point the (deformed) sandpile would be able to resist the extra force. Note that such an argument relies on the notion that applying the extra force will cause the material in contact with the segment that is pushed to move upwards, propagating the disturbance upwards without attenuation. Implicit in this argument is the idea that stresses can be related to strains; since the approach of Refs. [3] and [4] does not consider strains at all, it is not clear that the same argument would apply there, which is why we obtained a different experimental test (in the previous section).

Away from the angle of repose, the yield line in the outer plastic region in elastoplastic models tilts outwards from the vertical, at an angle that can be calculated in terms of the angle of the pile and the angle of repose. The above argument would apply there too, with the obvious modification that the extra applied force would have to be oriented along the yield direction.

\section{Experimental Consequences}

We now examine the effect on experiment of various approximations we have made: setting the Bagnold hysteresis angle to zero, applying an infinitesimal force to a perfectly continuous medium, and considering a twodimensional sandpile.

Real granular materials do not have a well defined angle of repose. If one pours grains slowly from a point source, the pile slowly builds up to a maximum angle; adding any more grains causes an avalanche in which the pile suddenly collapses to an angle that is about ten per cent less. If one keeps adding grains beyond this, the pile builds up once again to its maximum angle. The Bagnold hysteresis angle is the difference between the two angles before and after the avalanche. We have treated the angle of repose as determining the maximum shear stress that any part of the pile, regardless of its past history, can ever sustain. One should therefore build up the pile as close as possible to its maximum angle before putting on the perturbing force. Since the pile will never be exactly at its maximum angle, one would always need a finite force to destabilize it. However, a sufficiently large force applied anywhere in the pile will always destabilize it, so that identifying a region of incipient failure becomes difficult.

One can resolve this issue statistically: if the pile is repeatedly brought close to its maximum angle, and a small force is applied somewhere in the region of incipient failure, the probability of destabilizing the pile should be independent of where the force is applied. One should be able to detect the position of the line separating the stable region from the outer, unstable, region by applying the same magnitude perturbation at different distances from the surface. Throughout the outer region, the perturbation should be equally likely to make the pile fail. As soon as the position of the perturbation crosses into the inner region, the perturbation will become progressively less likely to cause failure as the distance from the separating line increases. Thus, if only a thin boundary layer is on the verge of flowing, the probability of destabilizing the pile will decrease rapidly when the point of application of the force is moved away from the surface.

The applied force should also be sufficiently small so as not to destabilize a region well below the Mohr-Coulomb yield point; it is preferable to distribute the force over several grains, since a highly localized force will produce much greater stresses in its vicinity. Finally, if one constructs a two-dimensional pile by pouring grains between parallel plates, the friction between the plates and the grains should be sufficiently small so as to avoid shear forces at the boundary plates stabilizing the pile.

These issues are much less important for elastoplastic theories, where the extra force is applied across an extended segment of the bottom plate, and the experiment can be performed even away from the maximum angle of repose. Of course, one would need to know the angle of the pile quite accurately in order to apply the perturbing force in the correct direction. 
In conclusion, we have shown that the existence of a wide failure zone adjoining the surface of a twodimensional sandpile built to the angle of repose is amenable to experimental verification. The presence of such a zone is perhaps surprising. That it should be predicted by two very different theories is remarkable and deserves a careful experimental study.

Acknowledgments We thank Michael Cates, Joe Goddard, Dov Levine and Tom Witten and for many stimulating and enlightening discussion about plasticity and fragility in sandpile models. The authors are grateful to the Institute for Theoretical Physics at Santa Barbara for their hospitality where this work was initiated. SRN was supported by the NSF under Award CTS-9710991 and by the MRSEC Program of the NSF under Award DMR-9400379. ON was supported by the Alfred P. Sloan Foundation.

[1] C.-H. Liu, S.R. Nagel, D.A. Schecter, S.N. Coppersmith, S.N. Majumdar, O. Narayan and T.A. Witten, Science
269, 513 (1995); S.N. Coppersmith, C.-H. Liu, S. Majumdar, O. Narayan and T.A. Witten, Phys. Rev. E53, 4673 (1996).

[2] F. Cantelaube and J.D. Goddard, in "Powders and Grains 97", R.P. Behringer and J.T. Jenkins eds. (Balkema 1997).

[3] J.-P. Bouchaud, M.E. Cates and P.J. Claudin, J. Phys. II France 5, 639 (1995).

[4] J.P. Wittmer, M.E. Cates and P. Claudin, J. Phys. I France 7, 39 (1997).

[5] H. M. Jaeger, S. R. Nagel, and R. P.Behringer, Rev. Mod. Phys. 68, 1259 (1996).

[6] Both types of theories (Refs. [2] and [3]) ignore the Bagnold hysteresis angle. This means that in those theories a pile can be built up to an angle of repose, and any material added subsequently will simply cause the pile to grow laterally, keeping the sandpile slope fixed at the angle of repose. In fact, the motion of the grains in the boundary layers lowers the inclination of the sandpile by the Bagnold hysteresis angle. Since this is approximated to zero in both approaches, one would expect the boundary layer to be of negligible width to within the same approximation. The implications for experiment of this assumption will be considered later.

[7] This can be achieved by choosing the perturbation $f$ to be small, and rounding it out so that it is not concentrated at a point. 